\title{
RESULTS OF THE 2005 INTERNATIONAL BUTTERFLY COUNTS IN SASKATCHEWAN
}

MIKE GOLLOP, 51 Welker Crescent, Saskatoon, SK S7H $3 M 3$ and ANNA LEIGHTON, 328 Saskatchewan Crescent West, Saskatoon, SK S7M OA4.

The Xerxes Society began a program of organized butterfly counts in 1974 . These counts were turned over to the North American Butterfly Association to administer beginning in 1993. Held within the month before or after July 4 , they are referred to as Fourth of July Counts $(4 \mathrm{JC})$ in the United States and First of July Counts (1JC) in Canada. The first of these counts was held in Saskatchewan in 1986 and the longest running count, the one at Fort Qu'Appelle, has been conducted annually since 1990 . Modeled on the Christmas Bird Count, each butterfly count is held within a $24 \mathrm{~km}$ circle and all individuals and species seen on

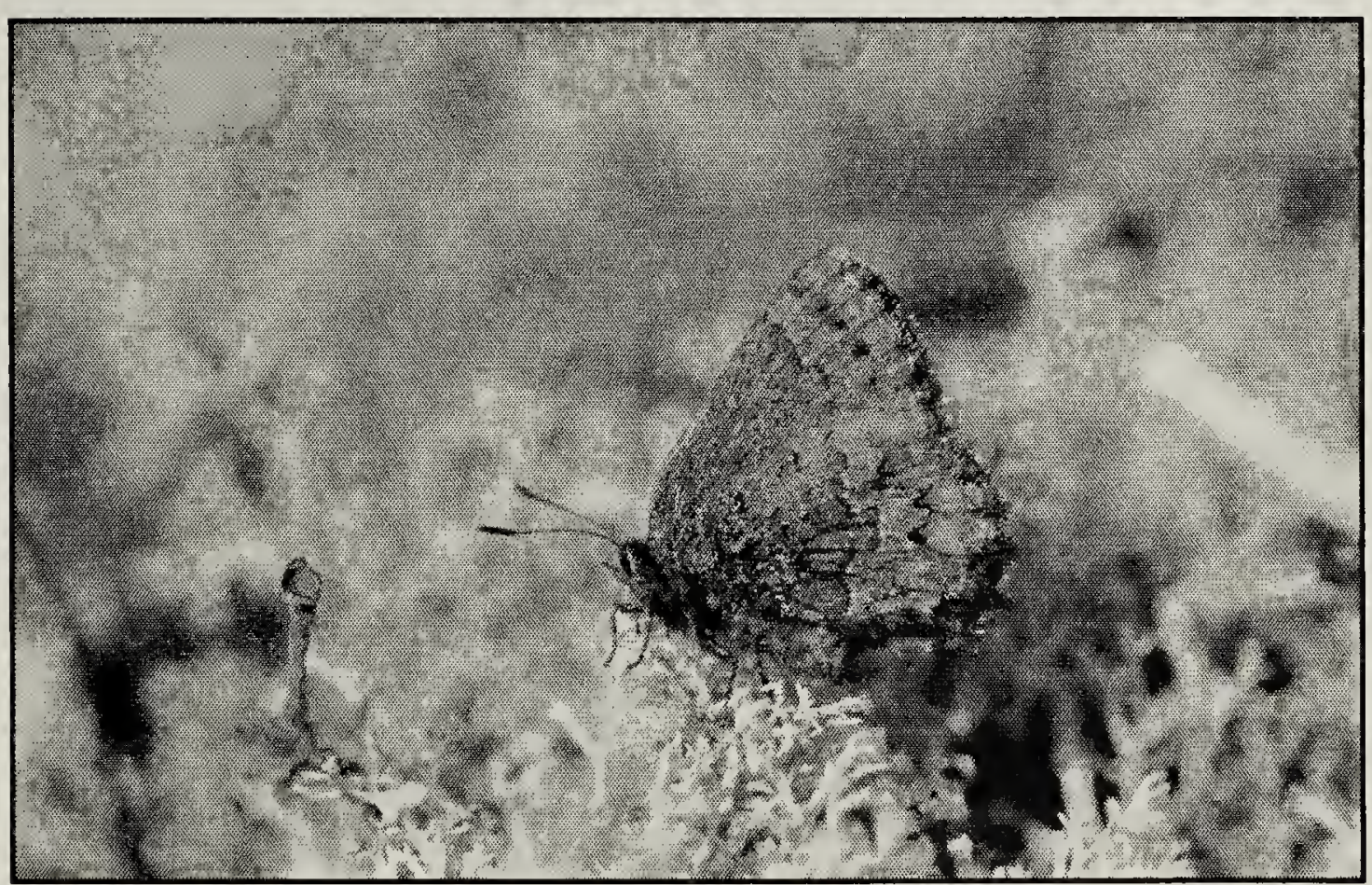

Figure 1. Eastern Pine Elfin on Waskesiu River count, 8 June 2005 count day are recorded. Unlike the Christmas Bird Count, the butterfly count day may be rescheduled if the weather is unfavorable for the target species; butterflies simply don't fly when the weather is rainy or cold.

In 2005, annual butterfly counts were conducted at eight locations in Saskatchewan: Dundurn Military Reserve, Fort Qu'Appelle, Nisbet Forest, Regina, Saskatoon, Preeceville, Waskesiu River and Woodpile Creek. This is fewer counts than in the past few years: 14 locations in 2004 and 18 in 2003. The reduced number of counts resulted in a total of 57 species counted in 2005 as compared to 79 in 2004 and 84 in 2003 . The total number of butterflies counted was 6961, however, compared to 4942 in 2004 and 9634 in 2003. Butterflies per party-hour averaged 86 on the eight counts, which was up from previous 
averages of 45 in 2004 and 68 in 2003. However, if the single count of 4788 Painted Ladies at Regina is omitted from these calculations, the average butterflies per party-hour for 2005 is only 27.

Participation has remained high in the last few years, with 33 different observers participating in the eight counts in 2005 . They clocked 81 partyhours and covered $129 \mathrm{~km}$ on foot and an additional $135 \mathrm{~km}$ by vehicle. Count conditions ranged from a low temperature of $11^{\circ} \mathrm{C}$ at Waskesiu River in the morning to highs of $32^{\circ} \mathrm{C}$ at Waskesiu River in the afternoon and at Woodpile Creek. (Table 1) The high temperature at Woodpile Creek was also accompanied by a mosquito flight that was comparable only to that of the northern tundra!

The number of species per count ranged from 10 at Woodpile Creek to 25 at Fort Qu'Appelle. Species totals were generally lower than average for the second year in a row for counts with at least a five-year history. This may reflect the cool moist conditions during these two years. For the eight counts conducted in all three years from 2003 to 2005 , the species totals have ranged from a low of 6 at Woodpile Creek in 2004 to a high of 35 at Preeceville in 2003 (which had 23 this year).

In 2005, the number of individual butterflies per count ranged from 36 at Woodpile Creek to 5034 at Regina. For the eight counts conducted in all three years from 2003 to 2005 , the total number of butterflies has ranged from as low as 8 at Woodpile Creek in 2004 to the 5034 reported from Regina in 2005.

Painted Ladies migrated into the province in large numbers in early to mid-June. Early counts recorded few if any of this species: Waskesiu River, on June 8 , recorded one Painted Lady while Dundurn did not have any on June 13. All other counts recorded Painted Ladies including exceptional counts of 4788 at Regina on June 22 and 473 at Saskatoon on July 9. (Table 2)

Other unusually high counts of species included 6 Christina Sulphurs at Nisbet Forest, 205 Spring Azures at Waskesiu River and 113 Common Ringlets at Regina. A common butterfly not recorded on the 2005 counts was the Meadow Fritillary. There were also no Gray Commas.

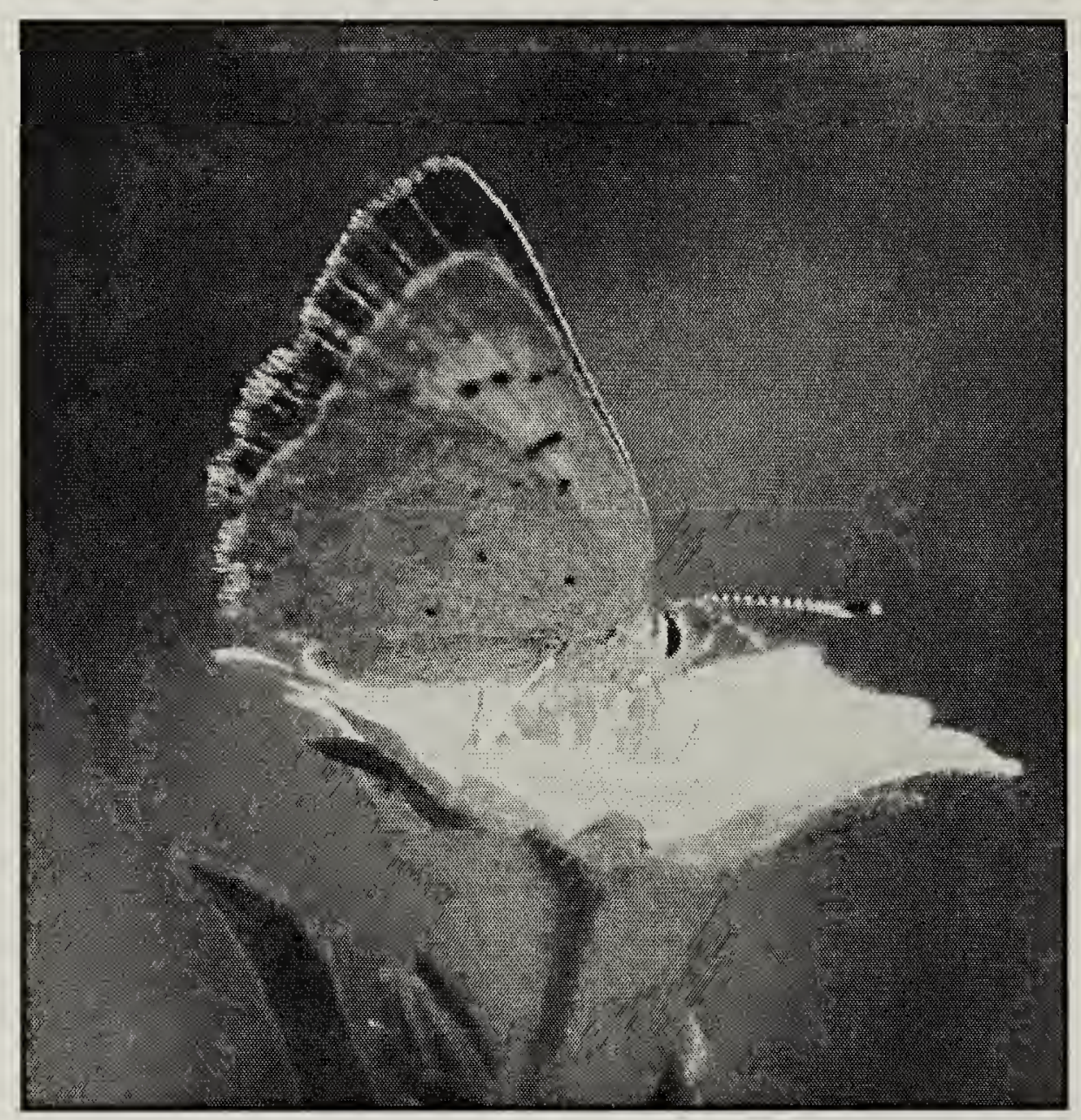

Figure 2. Dorcas Copper on host plant, Shrubby Cinquefoil. Nisbet Forest, 6 August 2005 
There were no provincially rare species recorded this year, although the Northern Blue at Fort Qu'Appelle is the first $1 \mathrm{JC}$ record, perhaps indicating that Ron Hooper is one of the few people with sufficient expertise to identify this challenging species! Four Dorcas Coppers at Nisbet Forest were also a good find. (Figures 2 and 3 ).

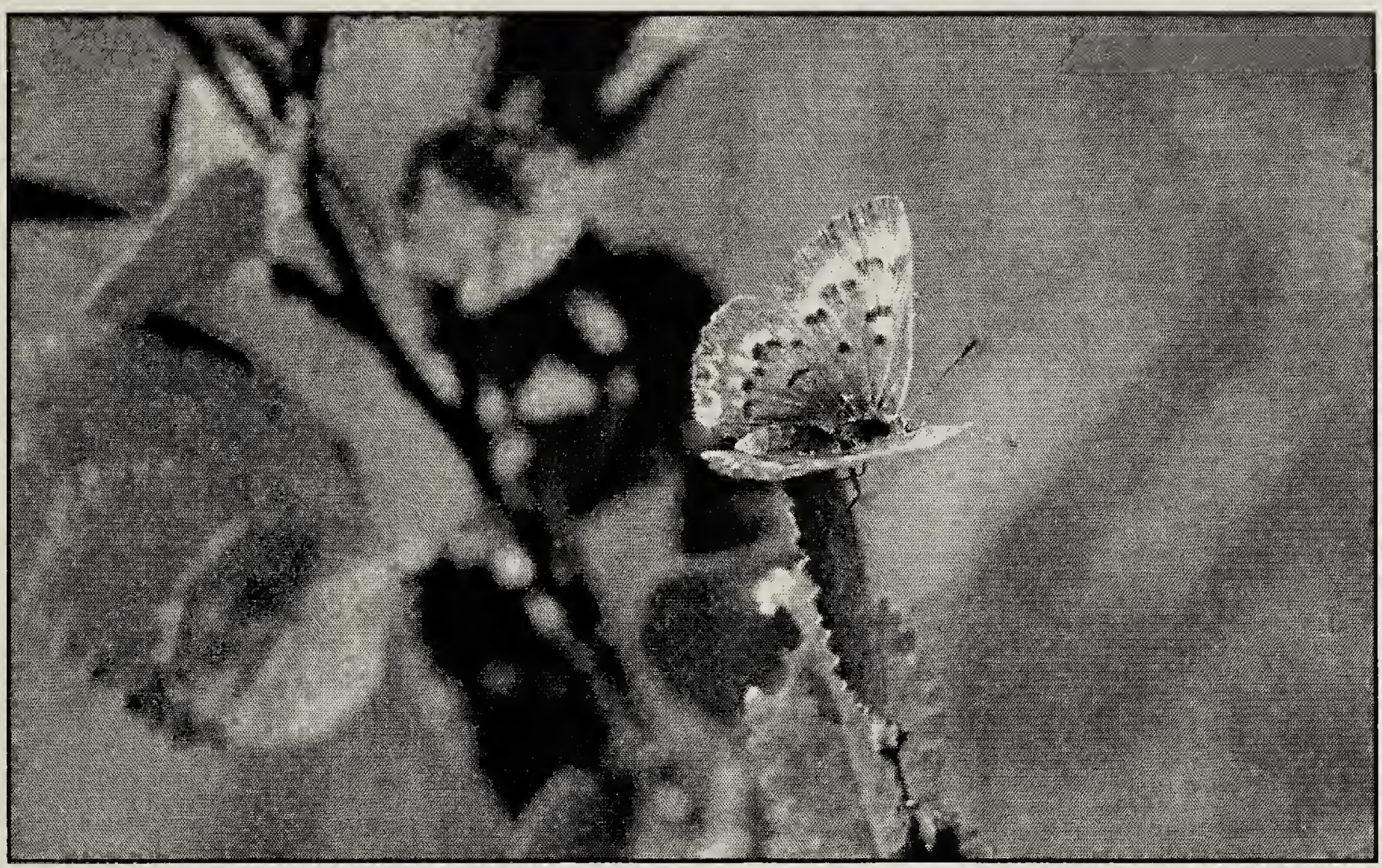

Figure 3. Dorcas Copper, female, dorsal surface. Nisbet Forest, 6 August 2005 Juhachi Asai

Table 1. 1JC Statistics - Saskatchewan 2005

\begin{tabular}{|c|c|c|c|c|c|c|c|c|c|c|c|c|c|}
\hline $\begin{array}{l}\text { 1JC } \\
\text { name }\end{array}$ & $\begin{array}{l}1.1 \mathrm{C} \\
\text { date } \\
\end{array}$ & $\begin{array}{l}\text { no. of } \\
\text { spp. }\end{array}$ & $\begin{array}{l}\text { no. of } \\
\text { b'flies }\end{array}$ & $\begin{array}{l}\text { b'flies/ } \\
\text { ph }\end{array}$ & $\begin{array}{l}\text { no. of } \\
\text { obs. }\end{array}$ & $\begin{array}{l}\text { no. of } \\
\mathrm{ph}\end{array}$ & $\begin{array}{l}\text { ph on } \\
\text { foot }\end{array}$ & $\begin{array}{l}\mathrm{km} \text { on } \\
\text { foot }\end{array}$ & $\begin{array}{l}\mathrm{km} \text { by } \\
\mathrm{car}\end{array}$ & time & $\begin{array}{l}\% \text { sun } \\
\text { am:pm }\end{array}$ & $\begin{array}{l}\text { temp. } \\
\text { deg.C }\end{array}$ & $\begin{array}{l}\text { wind } \\
\mathrm{km} / \mathrm{hr}\end{array}$ \\
\hline$\overline{\text { WaR }}$ & Jun 8 & 17 & 316 & 32.5 & 3 & 9.7 & 9.7 & 15 & 0 & $1030-1740$ & $0: 80$ & $11-32$ & light \\
\hline Dun & $\ln 13$ & $\overline{12}$ & 119 & 31 & 1 & 3.8 & 3.8 & $\overline{12}$ & 0 & $0920-1440$ & 90:90 & $19-25$ & $\overline{16}$ \\
\hline Reg & Jun 22 & 15 & 5034 & 479 & 8 & 10.5 & 9.51 & 14.5 & 13 & $0830-1500$ & $100: 100$ & $22-31$ & $8-32$ \\
\hline$\overline{\text { WOC }}$ & $\operatorname{Jun} 22$ & 10 & 36 & 8.2 & 1 & 4.4 & 4.4 & 20 & 0 & $0925-1800$ & $100: 100$ & $26-32$ & 16 \\
\hline$\overline{\mathrm{F}+\mathrm{Q}}$ & $\operatorname{Jun} 25$ & 25 & $\overline{193}$ & 32 & 2 & 6 & 5 & 8 & 56 & $0930-1600$ & $90: 75$ & $12-19.5$ & $\overline{24-40}$ \\
\hline Sto & jul 9 & 23 & 677 & 25 & 12 & 27.4 & 17.4 & 24 & 46 & $0930-1735$ & $75: 90$ & $23-28$ & $14-25$ \\
\hline Pre & Jul 16 & 23 & 395 & 33 & 4 & 12 & 11 & 12.8 & 20 & $0915-1700$ & $25: 75$ & $17-25$ & $32-48$ \\
\hline $\mathrm{NiF}$ & Aug 3 & 17 & 191 & 27 & 2 & 7 & 7 & 23 & 0 & $1000-1700$ & $50: 50$ & $15-25$ & $15-30$ \\
\hline totals & $8 \mathrm{IJCS}$ & 57 & 6961 & 86 & $\overline{33}$ & 80.8 & 67.8 & 129.3 & 135 & $0830-1806^{*}$ & & $11-33^{*}$ & $\overline{5-48^{*}}$ \\
\hline
\end{tabular}

Abbreviations used in the table: $\mathrm{WaR}=$ Waskesiu River, Dun = Dundurn Military Reserve, Reg $=$ Regina, FtQ $=$ Fort Qu'Appelle, $\mathrm{WoC}=$ Woodpile Creek, Sto $=$ Saskatoon, $\mathrm{NiF}=$ Nisbet Forest, Pre $=$ Preeceville, obs. $=$ observers, ph $=$ party hours, spp. $=$ species, ${ }^{*}$ extremes for the year. 


\section{Table 2. IJC results - Saskatchewan 2005}

\begin{tabular}{|c|c|c|c|c|c|c|c|c|c|}
\hline SPECIES NAME & $\begin{array}{l}\text { WaR* } \\
8 \mathrm{JN}\end{array}$ & $\begin{array}{r}\text { Dun } \\
13 \mathrm{JN}\end{array}$ & $\begin{array}{r}R e g \\
22 \mathrm{JN} \\
\end{array}$ & $\begin{array}{l}\text { WoC } \\
22 \mathrm{JN}\end{array}$ & $\begin{array}{r}\mathrm{FtQ} \\
25 \mathrm{JN} \\
\end{array}$ & $\begin{array}{l}\text { Sto } \\
9 \mathrm{JY}\end{array}$ & \begin{tabular}{|c} 
Pre \\
$16 \mathrm{JY}$ \\
\end{tabular} & $\begin{array}{r}\mathrm{NiF} \\
3 \mathrm{AU} \\
\end{array}$ & Totals \\
\hline Silver-spotted Skipper & \multirow{3}{*}{$\begin{array}{l}2 \\
9\end{array}$} & & & & & \multirow{3}{*}{\begin{tabular}{r|}
$9 \mathrm{JY}$ \\
1
\end{tabular}} & & & 1 \\
\hline Northern Cloudywing & & & & & 1 & & & & 3 \\
\hline Duskywing & & & & & & & & & 9 \\
\hline Dreamy $\quad$ Duskywing & 22 & & & & & & & & 22 \\
\hline $\begin{array}{l}\text { Juvenal's Duskywing } \\
\text { Afranius Duskywing }\end{array}$ & & & & & & & & & \\
\hline $\begin{array}{ll}\text { Persius } & \text { Duskywing }\end{array}$ & 5 & 3 & & & & & & & 8 \\
\hline Grizzled Skipper & & & & & & & & & \\
\hline C. Checkered Skipper & & & 2 & 6 & 1 & 4 & & & 13 \\
\hline Common Sootywing & & & & & & & & & \\
\hline Unident. Skipper & & & & & & & & & \\
\hline Arctic Skipper & 1 & & & & 4 & & & & 5 \\
\hline Skipper & & & & & & & & & \\
\hline Skipperling & & & 2 & 5 & 4 & 1 & 5 & & 17 \\
\hline Skipper & & & & 5 & & & & & 5 \\
\hline C. Branded Skipper & & & & & & & & & \\
\hline Plains Skipper & & & & & & & & & \\
\hline Dakota & & & & & & & & & \\
\hline Nevada & & & & & & & & & \\
\hline Skipper & & & & & & 1 & & & 1 \\
\hline Skipper & & & & & & & & & \\
\hline Tawny-edged Skipper & & & & & & & & & \\
\hline Long Dash Skipper & & & & & 1 & 4 & 2 & 1 & 8 \\
\hline Rhesus Skipper & & & & & & & & & \\
\hline Delaware Skipper & & & & & & & & & \\
\hline Woodland Skipper & & & & & & & & & \\
\hline Hobomok Skipper & & & & & 3 & & & & 3 \\
\hline Skipper & & & & & & & & 3 & 3 \\
\hline Skipper & & & & & & & & & \\
\hline Oslar's Roadside Skipper & & & & & & & & & \\
\hline Com. Roadside Skipper & & 1 & & & 4 & & & & 5 \\
\hline Old World Sw'tail (Dods) & & & & & & & & & \\
\hline Old World Sw'tail (Huds) & & & & & & & & & \\
\hline Anise $\quad$ Swallowtail & & & & & & & & & \\
\hline Anisel Old World Sw'tail & & & & & & & & & \\
\hline Can. Tiger Swallowtail & 3 & 4 & & & 4 & & & & 11 \\
\hline Unident. White & & & 6 & & & & 4 & & 10 \\
\hline Western White & & 4 & 14 & 1 & & 2 & & & 21 \\
\hline Margined & & & & & & & & & \\
\hline Mustard White & 2 & & & & & & & 2 & 4 \\
\hline Cabbage White & & & 4 & & 3 & 106 & 45 & 1 & 159 \\
\hline Large $\quad$ Marble & & & & & & & & & \\
\hline
\end{tabular}




\begin{tabular}{|c|c|c|c|c|c|c|c|c|c|}
\hline & WaR & Dun & Reg & WoC & $\mathrm{FtQ}$ & Sto & Pre & $\mathrm{NiF}$ & totals \\
\hline $\begin{array}{l}\text { Olympia Marble } \\
\text { Unident. Sulphur }\end{array}$ & & & & & & & & 5 & 5 \\
\hline Clouded Sulphur & & 9 & 25 & 2 & 7 & 9 & 4 & 9 & 65 \\
\hline Orange $\quad$ Sulphur & & & & & & & & & \\
\hline Q. Alexandra's Sulphur & & & & & & & & & \\
\hline Christina Sulphur & & & & & & & 6 & & 6 \\
\hline Giant $\quad$ Sulphur & & & & & & & & & \\
\hline $\begin{array}{l}\text { Pink-edged Sulphur } \\
\text { Harvester }\end{array}$ & & & & & & & & & \\
\hline Unident. Copper & & & & & & & 1 & & 1 \\
\hline Gray Copper & & & & & & & & & \\
\hline Copper & & & & & & & & & \\
\hline Copper & & & & & & & & & \\
\hline Dorcas & & & & & & & & 4 & 4 \\
\hline Purplish & & 1 & 24 & & & & & & 25 \\
\hline Acadian Hairstreak & & & & & & & & & \\
\hline Hairstreak & & & & & 1 & & & 1 & 2 \\
\hline Edwards' Hairstreak & & & & & & & & & \\
\hline Banded Hairstreak & & & & & & & & & \\
\hline Hairstreak & & & & & & & 1 & & 1 \\
\hline Unident. & 2 & & & & & & & & 2 \\
\hline Brown & 10 & & & & & & & & $\overline{10}$ \\
\hline Hoary & & & & & & & & & \\
\hline Eastern Pine Elfin & 3 & & & & & & & & 3 \\
\hline Western Pine Elfin & & & & & & & & & \\
\hline Gray Hairstreak & & & & & & & & & \\
\hline Unident. Blue & 19 & 1 & 5 & & & 2 & 3 & & 30 \\
\hline West. Tailed Blue & 3 & 3 & & & 10 & $\overline{1}$ & $\overline{14}$ & & 31 \\
\hline Spring Azure & 205 & & & & & & & & 205 \\
\hline Summer Azure & & & & & & & & & \\
\hline Rocky Mt.Dotted Blue & & & & & & & & & \\
\hline Arrowhead Blue & & & & & & & & & \\
\hline Silvery Blue & 10 & 2 & 45 & & 7 & 4 & 4 & & 72 \\
\hline Northern Blue & & & & & 1 & & & & 1 \\
\hline Melissa Blue & & 3 & 4 & & 5 & 1 & & & 13 \\
\hline Greenish Blue & & 1 & 5 & & 7 & 4 & 6 & & 23 \\
\hline Boisduval's Blue & & & & & & & & & \\
\hline Lupine (Acmon) Blue & & & & & & & & & \\
\hline Prairie Arctic Blue & & & & & & & & & \\
\hline Unident. large Fritillary & & & & & & 6 & 9 & 5 & 20 \\
\hline Variegated Fritillary & & & & & & 1 & 1 & 1 & 3 \\
\hline Great Spangled Frit. & & & & & 1 & 5 & & 1 & 7 \\
\hline
\end{tabular}




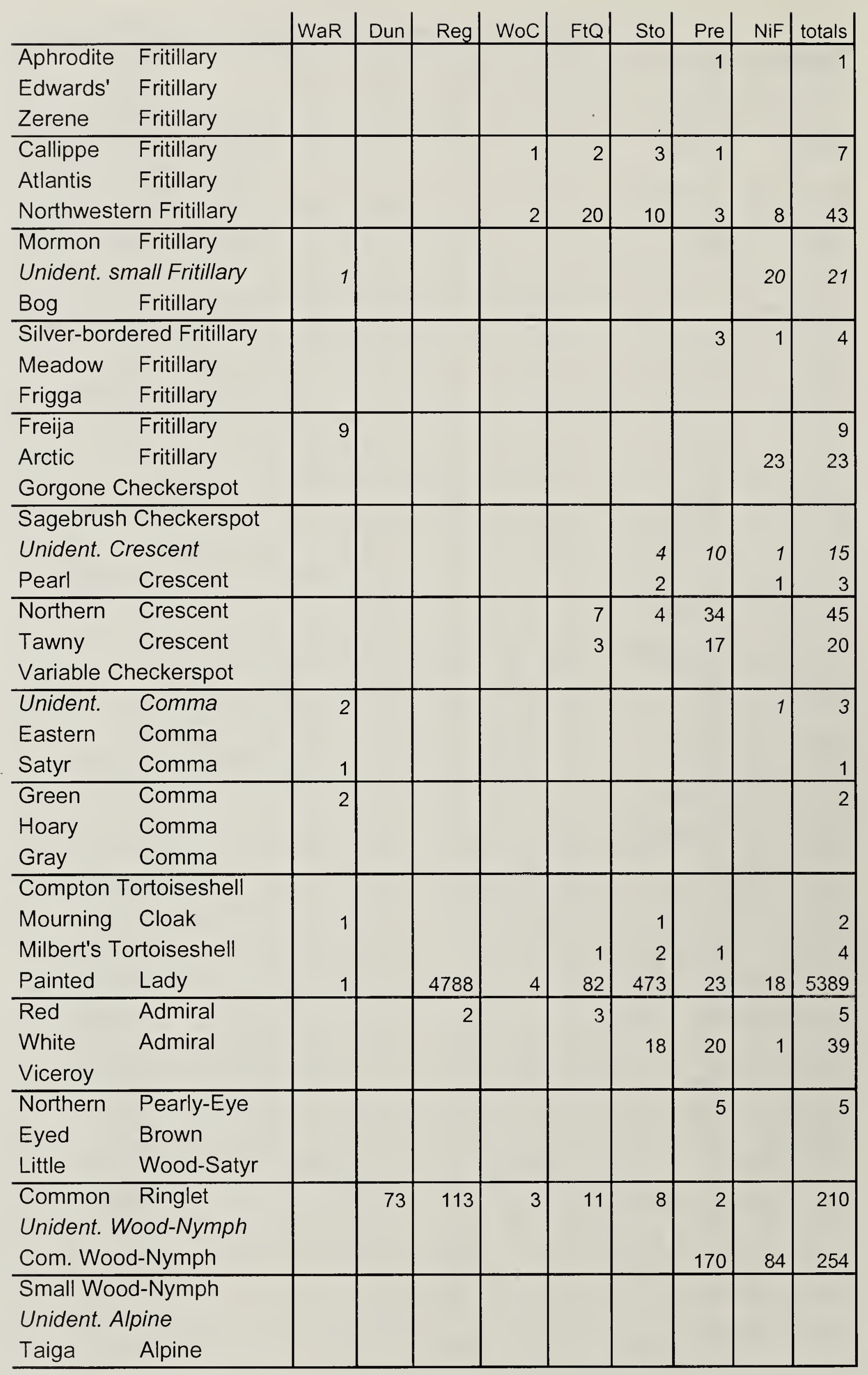




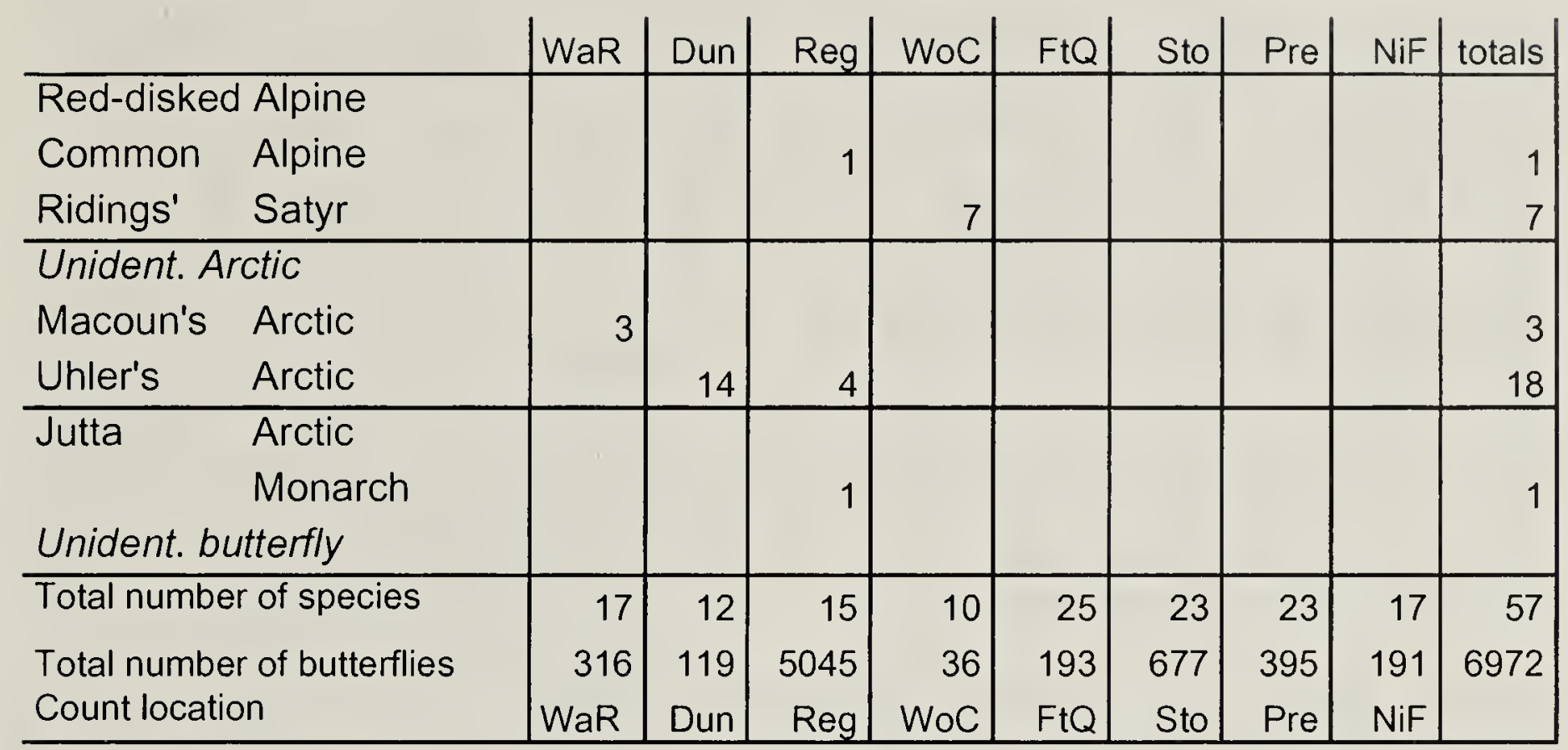

${ }^{*} \mathrm{WaR}=$ Waskesiu River, Dun $=$ Dundurn Military Reserve, Reg $=$ Regina, FtQ = Fort Qu'Appelle, $\mathrm{WoC}=$ Woodpile Creek, Sto $=$ Saskatoon, NiF $=$ Nisbet Forest, Pre $=$ Preeceville.

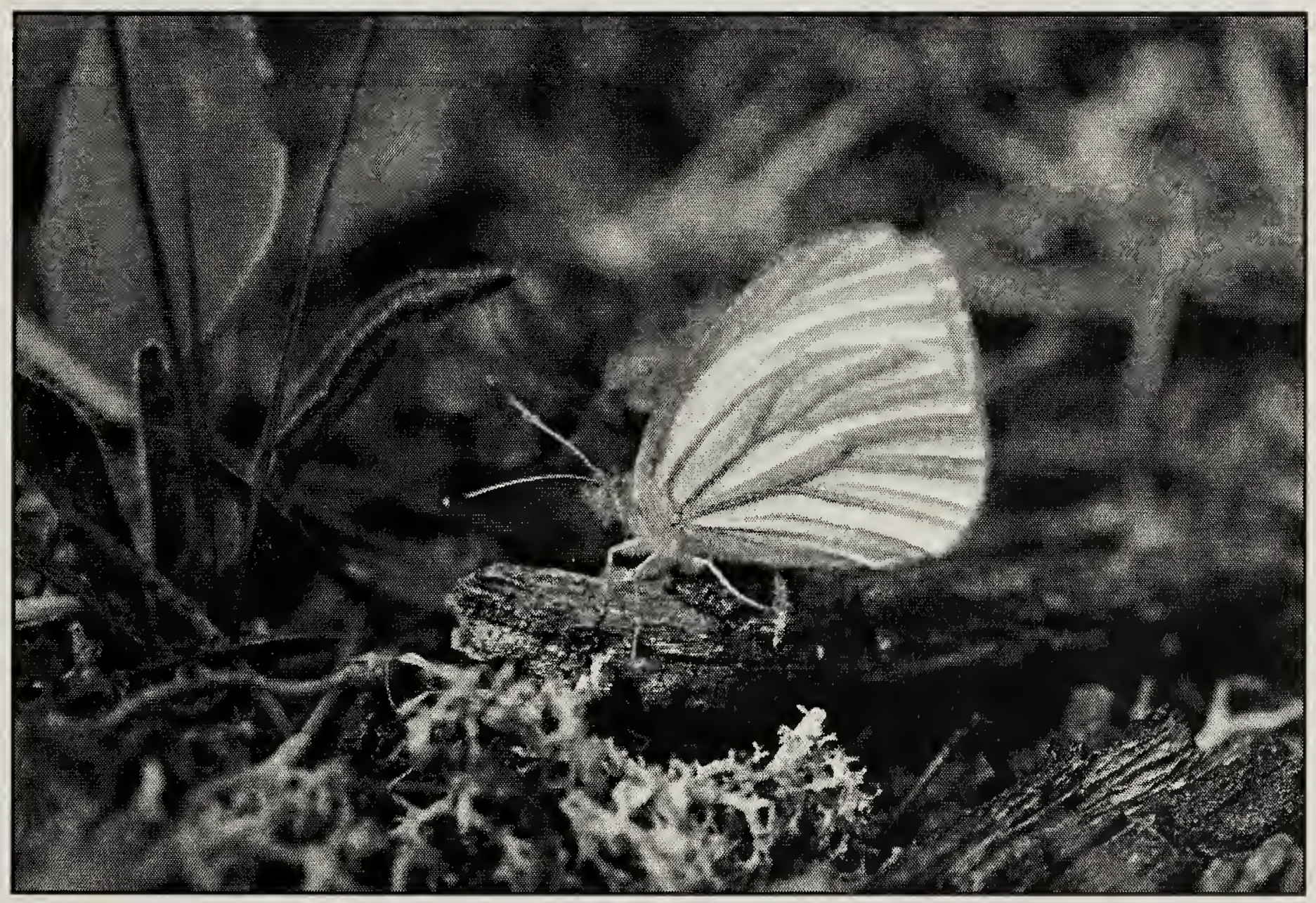

Figure 4. Mustard White on Waskesiu River count, 8 June 2005

Juhachi Asai 